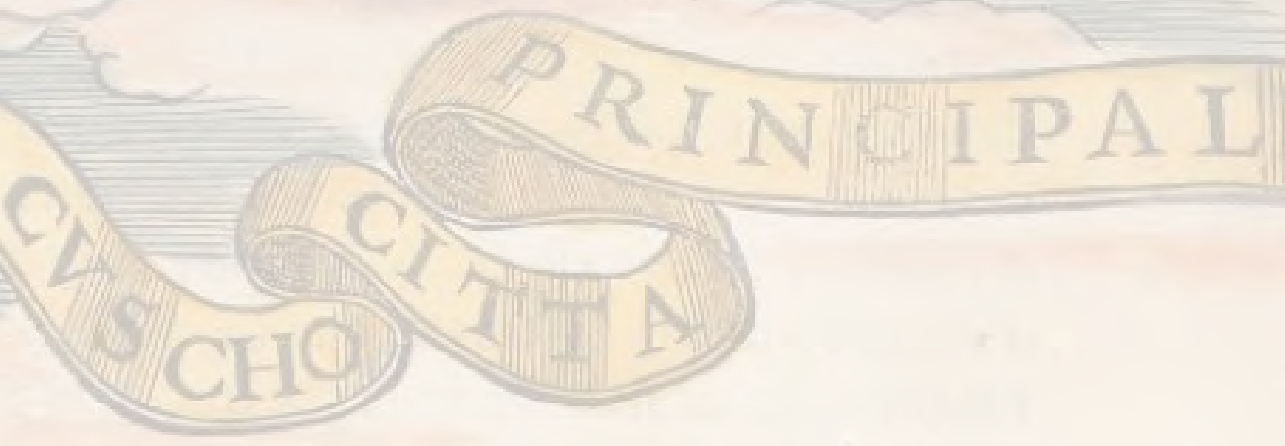

\title{
La Universidad Andina del Cusco
}

En el Cusco, por iniciativa de cusqueños motivados por brindar una alternativa de educación superior a la juventud, que en ese tiempo sólo contaba con una universidad pública, el 5 de octubre de 1979 se conformó la sociedad civil, promotora de la UNIVERSIDAD ANDINA DEL CUSCO, integrada por los doctores Antonio Callo Cáceres, Néstor Bustos Silva, Cesar Cornejo Foronda y Monseñor Luis Vallejos Santoni, posteriormente convertida en la Asociación Civil Promotora de la Universidad Andina del Cusco.

Se logró su creación oficial, el 23 de mayo de 1984, mediante la Ley Nro. 23837, "Ley de creación de la Universidad Andina del Cusco". A partir de ese momento, la Universidad Andina empieza a encaminar una labor de servicio a la comunidad cusqueña con siete carreras profesionales, contando con la voluntad de sus autoridades, docentes, personal administrativo y, sobretodo, con la confianza de sus estudiantes, empezó a caminar con pasos firmes, forjando su propia personalidad y consolidando la confianza de la comunidad cusqueña, a la que hoy se debe.

Cuando hablamos de la personalidad de la Universidad Andina del Cusco, nos referimos a que cuenta con un modelo propio educativo, denominado "Modelo Antropológico Filosófico del Paradigma Trascendental”, un modelo en el cual se conjugan valores occidentales y valores andinos en una suerte de interculturalidad diferente a como se ha enfocado en otros espacios educativos, marcando la diferencia en educación superior. Han transcurrido treinta y cinco años desde su creación, la Universidad Andina vuela cual la ave andina milenaria, el cóndor apuchín, que la representa como su símbolo señero; hoy cuenta con una población estudiantil de dieciocho mil alumnos, con más de setecientos sesenta y seis docentes, ciento setenta y tres jefes de práctica y doscientos sesenta y dos colaboradores administrativos, albergados en su sede central universitaria del Cusco, Q'ollan y en sus filiales. Académicamente se compone por cinco facultades: i) la Facultad de Ciencias Económicas Administrativas y Contables, con seis escuelas profesionales (Administración, Economía, Contabilidad, Marketing, Negocios Internacionales y Finanzas; ii) la Facultad de Ingeniería y Arquitectura con cinco escuelas profesionales (Ingeniería Industrial, Ingeniería de Sistemas, Ingeniería Civil, Ingeniería Ambiental y Arquitectura; iii) la Facultad de Ciencias y Humanidades con dos escuelas profesionales (Educación Inicial - Primaria y Turismo, teniendo a

POR ANA CELIA CHÁVEZ CHACÓN. Licenciada especialista profesional de Licenciamiento. Universidad Andina del Cusco. achavez@uandina.edu.pe 


\section{ville Capitale du Royaume de Peru.}

Estudios Generales bajo su cargo y, iv) la Facultad de Derecho en la sede Central de Cusco.

En el local de Qóllana se encuentra la Facultad de Ciencias de la Salud, con seis escuelas profesionales: Psicología, Medicina humana, Estomatología, Enfermería, Obstetricia y Tecnología Médica, con mención rehabilitación física.

La Universidad Andina también cuenta con tres filiales, una en el distrito de Sicuani, provincia de Canchis, con tres escuelas profesionales: Administración, Derecho y Contabilidad; la otra en la ciudad de Quillabamba, provincia de La Convención y la tercera en Puerto Maldonado, en el departamento de Madre de Dios, con las mismas escuelas profesionales de la primera filial.

A nivel de Posgrado, cuenta con una Escuela con dieciséis maestrías y nueve doctorados, albergando, actualmente a más de seiscientos ochenta y dos estudiantes, cincuenta y cuatro docentes invitados, que desarrolla su actividad administrativa y académica en un local que se encuentra en el centro histórico del Cusco.

El equipamiento tecnológico de la universidad es moderno, con pizarras interactivas y laboratorios de última generación, ubicándose a la vanguardia de la formación superior de calidad, con avances en las ciencias de la salud en plastinación de órganos humanos, simuladores y el bioterio que dan soporte a las investigaciones.

Se está avanzando a pasos grandes en investigación, con publicación de artículos y trabajos científicos con relevancia social. La responsabilidad social es otro eje principal de la universidad, que se articula con diversas empresas, afiliando los trabajos de investigación con un fin de proyección hacia la comunidad.
Actualmente la responsabilidad directiva de la universidad, está a cargo de autoridades competentes, lideradas por su Rector, el Dr. Emeterio Mendoza Bolívar; la Vicerrectora Académica, Dra. Daisy Núñez del Prado Béjar; la Vicerrectora de Investigación, Dra. Di Yadira Bravo Gonzales y, la Vicerrectora Administrativa, la Dra. María Antonieta Olivares Torre, quienes están logrando la internacionalización y la presencia de la Universidad Andina en el mundo, con un especial énfasis en una política de calidad, obteniendo la acreditación internacional con la RIEV, y logrando el Licenciamiento institucional por la SUNEDU, colocando a la Universidad Andina del Cusco como líder local, interregional e internacionalmente mediante los convenios bilaterales con países a nivel mundial. 


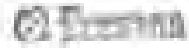

CREAN CON EL CARACIER DE PRI. VADA LA UNIVERSIDAD ANDINA DEL CUSCO, CON SEDE EN LA CIU DAD DEL CUSCO COMO PERSONA IURIDICA DE DERECIIO PRIVADOORGANDADA POR LA ASOCLACION CIVIL PROMOTORA DE LA UNIVEHSIDAD PARTICULAR ANDINA DEL. CUSOO.

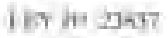

14 nevibers beta errumices

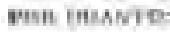

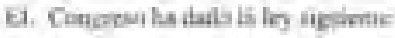

2L CONGRLSO DE LARENLIEA OLL FRU fit thit it an melime

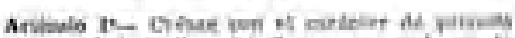

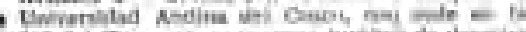

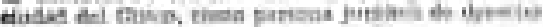

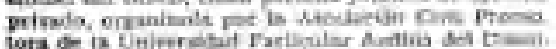

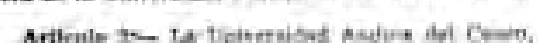

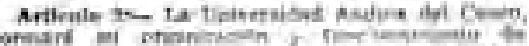

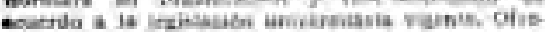

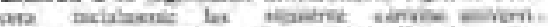

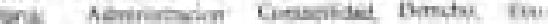

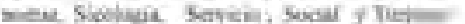

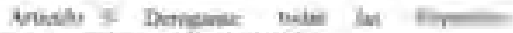

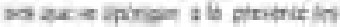

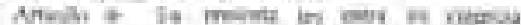

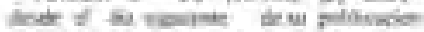

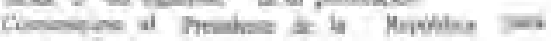
w monilorim

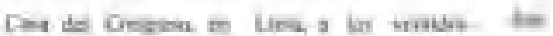

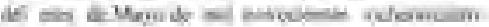

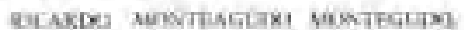

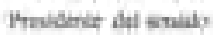

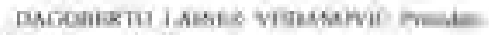

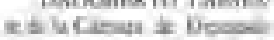

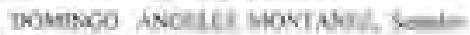
Sesisuma

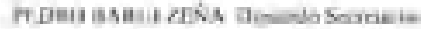

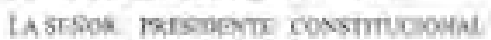
OE LA BINAWLE'

10in raster.

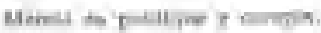

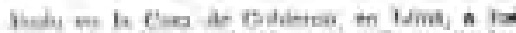

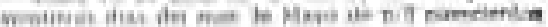
genilimation

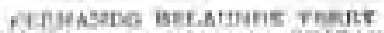
CAtratre Ramiarsa crimates

\section{Q2.2uterns}

MODIFICAN FE ARTICTLO 2 DE LA LEY No. 23837

LiV $3 w^{*} 24631$

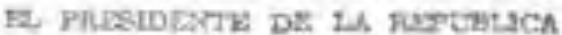
pon cunvio.

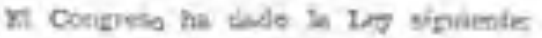
PE:0,

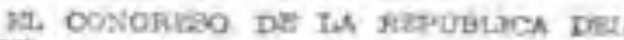

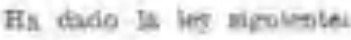

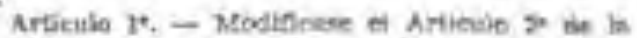

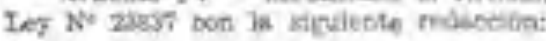

- Artíruís Y. - Ia Univereitad Privean An-

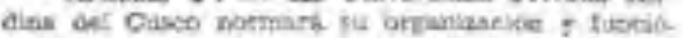
numiknte coen soleción a la batiblactón uriversite. na vigrale. Otreeerd, Iniclatmenie. las sirumies

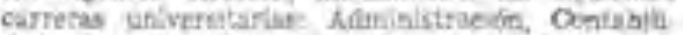

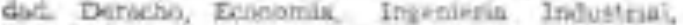

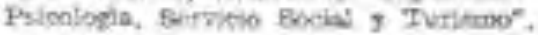

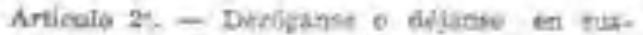

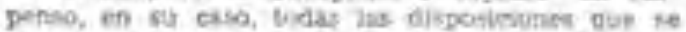
eponcan o la pretence ing, in eug rike dusde ni dis eigulente of wi guthinanite.

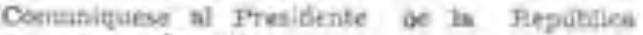
para sut promitimeide.

Cas del Congreso, to Itma a les dencisiete dias del mes de Dieletabre de mil buwedentos ochentiseis.

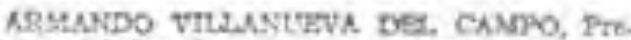
zidtenive des Smado

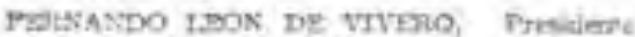
dp- Le Clmara de Dipatedes

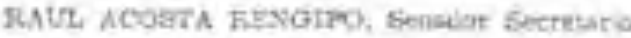

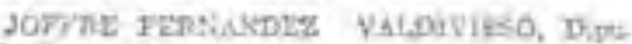
trás Eerretand

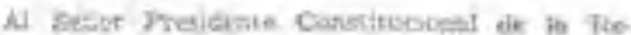
Dthata

Fote TANTQ:

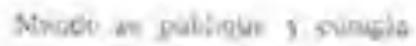

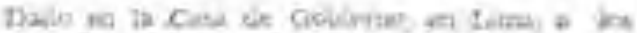

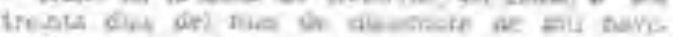
catcitos veriegrima

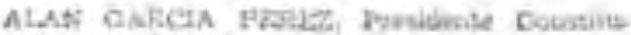
coow of In Forvibis.

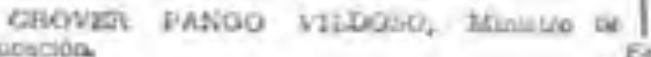


ASRMBLEA HACTONAL DE RECTOMES

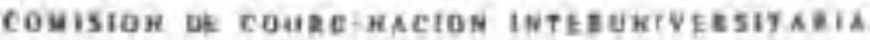

Resoludion tia. 195-92-AKR

Serket $\mathrm{Tr} \%$.

CARLOS GOMEZ ERLZA

Prosidente de Ia Comidión

Lina 50 do dtefeabre de 1992

Oryamiandara de la Uriverdida

Andint del Clasce

CUSCD.

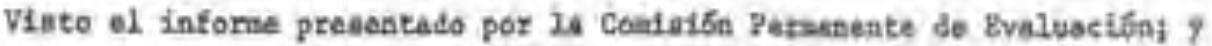

COSSTDERANDO

Que, In Heiveraidad Privada de1 Caseo fub arbada por Leg g"23037;

Que, is Dntveroidad acocionade be ventalo functorusolo desde su creacton basta

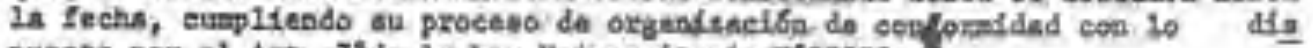

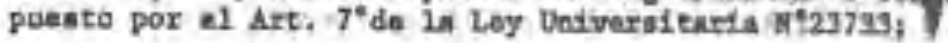

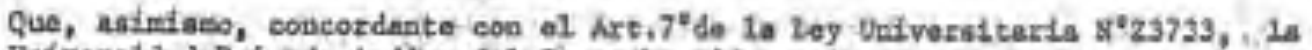

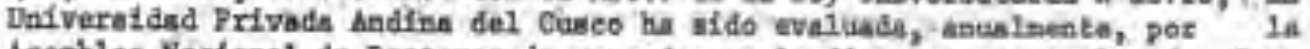

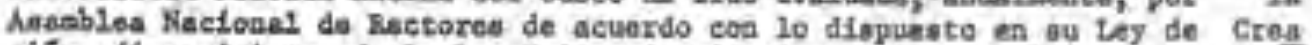

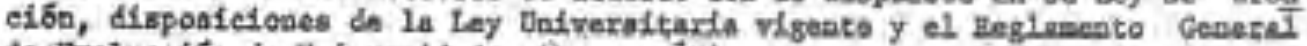

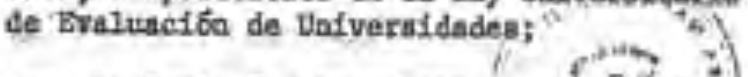

Que, In Condsión Pemanaste de Eviquféfisn

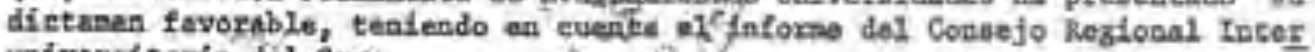
univereitrario dol sur;

Que, de Ine evaluscioneg realisadas oportuthatente ve deoprondo que Ia Univer aldad Privada Asdina del Canco ha culninado su proceeo de Organtaector den tro de los plazon legales previatos, $y$ ha desostrado que seline lad requjof too alninou de infreastructura, ostructura scedifica adecusda a is Lay de

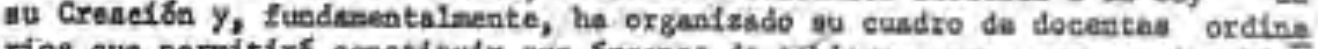
rioa que permitirá coastituly gus organqe de goblaxtan, onno nons hasmbles Dof veraitaris, Conaejo Univeraitarlo y Coasejo do Faculead;

Que, en conecuencis, procede otorgar autoriascibn da funcionaelento datinitivo a la Dniveraidad Privada Aadins del Cuaco, la mians que debazE danarro

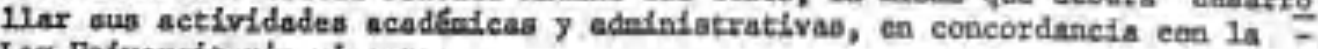
Leg Daiversitarin vigente;

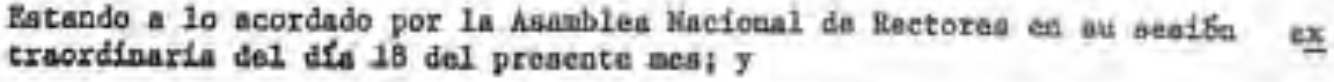

En uso de Ino atribeciones que ie competen a la haseblen Kactonal de Recto res en virtud de $1 \mathrm{~L}$ Ley Jufveraitaria $\mathrm{N}^{*} 23733$;

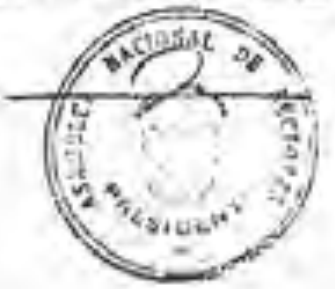

